\title{
Aqueous humour concentrations after topical apPlication of combinEd levofloxacin-dexamethasone eye dRops and of its single components: a randoMised, assEssor-blinded, parallel-group study in patients undergoing cataract surgery: the iPERME study
}

\author{
Michele Figus $^{1} \cdot$ Chiara Posarelli $^{1}$ - Dario Romano ${ }^{2} \cdot$ Marco Nardi $^{1} \cdot$ Luca Rossetti ${ }^{2}$
}

Received: 23 November 2019 / Accepted: 26 March 2020 / Published online: 13 April 2020

(C) The Author(s) 2020

\begin{abstract}
Purpose To evaluate the penetration of levofloxacin and dexamethasone sodium phosphate into the aqueous humour (AH) after administration in combination and as single molecules. Evaluation of the penetration of those agents in the site of action and their pharmacodynamic potential activity in view of the intended clinical use after cataract surgery.

Methods Randomised, assessor-blinded, parallel-group. Patients scheduled for cataract surgery were assigned in a 1:1:1 ratio to: levofloxacin + dexamethasone sodium phosphate (L-DSP), Levofloxacin (L) or Dexamethasone sodium phosphate (DSP) eye drops. Either test or reference drugs were instilled in the cul-de-sac twice, 90 and $60 \mathrm{~min}$ before paracentesis.

Results A total of 125 patients completed the study. Fraction of dose absorbed in the anterior chamber was $3.8-4.2 \cdot 10^{-4}$ for levofloxacin and $0.3-0.4 \cdot 10^{-4}$ for dexamethasone, respectively. No notable differences in concentration of levofloxacin were found between L-DSP $\operatorname{arm}(1.970 \mathrm{nmol} / \mathrm{ml})$ and $\mathrm{L}$ arm $(2.151 \mathrm{nmol} / \mathrm{ml})$. The concentrations of levofloxacin were well above the MICs for the most frequent Gram-positive and Gram-negative eye pathogens. Dexamethasone concentrations were slightly lower in L-DSP arm $(0.030 \mathrm{nmol} / \mathrm{ml})$ than in DSP arm $(0.042 \mathrm{nmol} / \mathrm{ml})$, but still in the pharmacodynamically active range in the site of action. The difference was not clinically relevant. DSP was not detected in any HA sample, suggesting its full hydrolysis to free dexamethasone. Conclusion Our results confirm that no interaction is evident on the corneal penetration of levofloxacin and dexamethasone which reach pharmacologically active concentrations when instilled as fixed combination eye drops to patients undergoing cataract surgery.
\end{abstract}

Trial registration ClinicalTrials.gov Identifier: NCT03740659

Keywords Levofloxacin $\cdot$ Dexamethasone $\cdot$ Combination eye drop $\cdot$ Aqueous humour

\section{Introduction}

Postoperative endophthalmitis is an inflammatory condition of the eye, due to an infectious process from bacteria, fungi

Electronic supplementary material The online version of this article (https://doi.org/10.1007/s00228-020-02863-7) contains supplementary material, which is available to authorized users.

Michele Figus

michele.figus@unipi.it

1 Department of Surgical, Medical, Molecular Pathology and of Critical Area, University of Pisa, Via Paradisa, 2, 56100 Pisa, Italy

2 Eye Clinic, ASST Santi Paolo e Carlo, University of Milan, Milan, Italy or, on rare occasions, parasites that enter the eye during the perioperative period [1]. After an initial incubation phase lasting 16-18 $\mathrm{h}$ to a few days, an acceleration phase and a destructive phase of the infection develop. The acceleration phase follows primary infection of the posterior segment and leads to inflammation of the anterior chamber [1].

The prophylaxis of postoperative bacterial ocular infections such as endophthalmitis consists in the administration of intraocular, usually intracameral or subconjunctival antibiotics during surgery. Both pre- and postoperative topical applications are also used frequently [2]. To increase efficacy, a broad-spectrum antibiotic is indicated, and topical use of quinolones may represent a rational choice given their bactericidal activity against both the gram-positive and gram-negative bacteria most frequently responsible for bacterial eye infections 
[3]. Postoperative care after cataract surgery consists of topical anti-inflammatory and antibacterial drug prophylaxis.

Eye Drop Combi (L-DSP) is a fixed dose combination (FDC) of eye drops solution, with $0.5 \%$ levofloxacin and $0.132 \%$ dexamethasone sodium phosphate (corresponding to $0.1 \%$ free dexamethasone) as active ingredients, under development by NTC Srl, Milan (Italy).

The pharmacokinetics [4-9] of either levofloxacin or dexamethasone administered individually confirm that after application as an eye drop composition, either active drug reaches significant $\mathrm{AH}$ concentrations, with peaks of aqueous levels between 90 and $150 \mathrm{~min}$, and detectable concentrations after $12 \mathrm{~h}$ from dosing. Animal studies confirmed that after eye instillation of L-DSP at the human doses and for a short period of time, no local or systemic toxicity is expected (NTC, data on file, 2019).

The primary objective of this study was to analyse the $\mathrm{AH}$ drug concentrations of levofloxacin and dexamethasone instilled in the cul-de-sac either in combination or as single components. Secondary objectives included the evaluation of the penetration of those agents in the site of action and their pharmacodynamic potential activity in view of the intended clinical use for postoperative prophylaxis in cataract surgery.

\section{Material and methods}

\section{Design}

This was a multicentre, randomised, assessor-blinded, parallel-group clinical study. Patients scheduled for cataract surgery from September 2018 until December 2018 were recruited at the Ophthalmic Unit both of Azienda Ospedaliero Universitaria Pisana (Centre 001) and at the Eye Clinic and Head and Neck Department, ASST Santi Paolo e Carlo Hospital, University of Milan (Centre 002).

Randomisation list, monitoring of clinical centres and data management and statistics were provided by OPIS, an EU independent, full service clinical Contract Research Organization (CRO) with HQ in Desio (MB, Italy). The randomisation list was generated at OPIS by a statistician not involved in the study through a validated SAS program (SAS® for Windows release 9.4 (64-bit)). The allocation ratio was $1: 1: 1$, and the randomisation was managed by means of an electronic system (Clinical.NET).

The study was full GCP and GLP. All procedures performed in the study involving human participants were in accordance with the ethical standards and with the 1964 Helsinki declaration and its later amendments. The protocol was approved by the institutional research committees of the investigating centres, i.e. Comitato Etico Regione Toscana Area Vasta Nord Ovest c/o Azienda Ospedaliero Universitaria Pisana via Roma, 67 - 56126 Pisa (PI) (approval letter on May 2, 2018) and Comitato Etico Milano Area 1 c/o ASST FBF Sacco - P.O. L. Sacco Via G.B. Grassi, 7420157 Milano (approval letter on September 9, 2018).

\section{Patients}

Informed consent was obtained from all patients for being included in the study. Main eligibility criteria included patients of both genders, aged $\geq 40$ years, and scheduled for phacoemulsification. Corneal thickness measured by pachymetry was between 450 and $600 \mu \mathrm{m}$, and corneal integrity was confirmed by means of fluorescein test. Patients must have an adequate pupil dilation. Patients without confirmed corneal epithelium integrity, with glaucoma or with history of ocular surgery, or corneal disease or dystrophy, or ocular trauma with corneal damage, or acute ocular inflammation in the previous 6 months were excluded. Also excluded were female patients of childbearing potential without a negative pregnancy test, patients under treatment with an ophthalmic investigational drug in the 3 months prior to screening or treated within $12 \mathrm{~h}$ before start of cataract surgery with any topical ocular drug other than study drugs and instillation of topical anaesthetic (oxybuprocaine hydrochloride) followed by povidone-iodine within $10 \mathrm{~min}$ before start of surgery, i.e. at least 80 and 50 min after the first and second drop, respectively). Finally, those patients treated with any topical or systemic steroid or antibiotic drug in the 7 days prior to cataract surgery were excluded, too.

At screening, which must take place not more than 28 days before planned surgery, information was collected concerning demographics, medical history and any use of concomitant medications. Pachymetry and fluorescein test were performed to measure corneal thickness and to confirm corneal epithelium integrity. Prior to surgery, a pre-dose visit was done to confirm eligibility criteria.

\section{Treatments}

Eligible patients were randomly assigned to one of the three following treatment arms in a 1:1:1 ratio via an Interactive Web Response System (IWRS) provided by OPIS (Fig. 1):

- Test drug: levofloxacin $5 \mathrm{mg} / \mathrm{ml}$ + dexamethasone sodium phosphate $1.32 \mathrm{mg} / \mathrm{ml}$ eye drops (L-DSP) ${ }^{1}$ two $30-\mu 1$ doses, $30 \mathrm{~min}$ apart, one $90 \pm 15 \mathrm{~min}$ prior to surgery and the other $60 \pm 15 \mathrm{~min}$ prior to surgery. Total dose instilled: levofloxacin $300.0 \mu \mathrm{g}(=830.17 \mathrm{nMoles})$ and dexamethasone sodium phosphate $79.2 \mu \mathrm{g}$ (= 153.37 nMoles).

\footnotetext{
${ }^{1}$ Levendex® NTC srl, Milan, Italy. Brands in other countries: Ducressa®, Sevendoc $®$
} 
Levofloxacin $5 \mathrm{mg} / \mathrm{ml}$ + Dexamethasone $1 \mathrm{mg} / \mathrm{ml}$

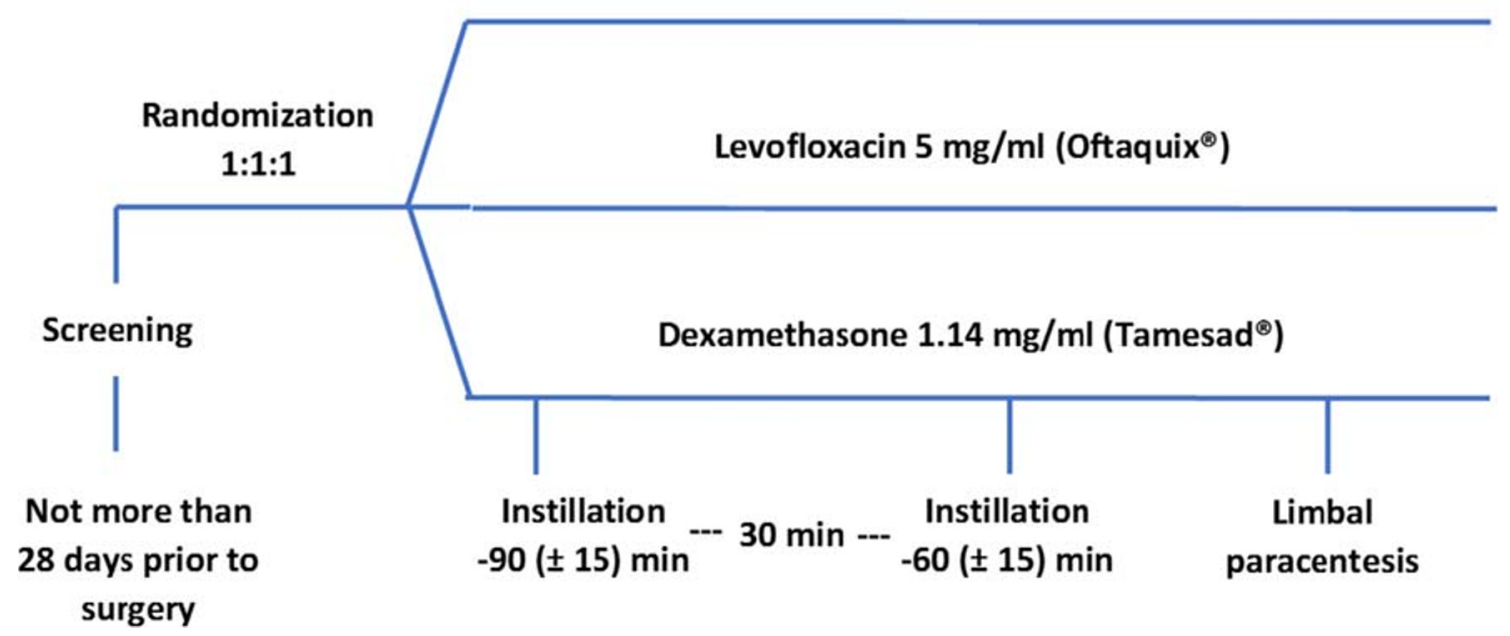

Fig. 1 Study design

- Reference A: levofloxacin $5 \mathrm{mg} / \mathrm{ml}$ eye drops (Oftaquix ${ }^{\circledR}$ ): two $30-\mu 1$ doses, $30 \mathrm{~min}$ apart, one $90 \pm$ $15 \mathrm{~min}$ prior to surgery and the other $60 \pm 15 \mathrm{~min}$ prior to surgery. Total dose instilled: levofloxacin $300.0 \mu \mathrm{g}$ (= 830.17 nMoles)

- Reference B: dexamethasone $1.5 \mathrm{mg} / \mathrm{ml}$ eye drops (Tamesad®, DSP): two 26- $\mu$ l doses, 30 min apart, one $90 \pm 15 \mathrm{~min}$ prior to surgery and the other $60 \pm 15 \mathrm{~min}$ prior to surgery. Total dose instilled: dexamethasone sodium phosphate $78 \mu \mathrm{g}$ (= $151.04 \mathrm{nMoles})$

\section{Procedure}

Administration of study drugs was performed by qualified health care personnel using a micropipette and dispenser. Doses were administered at the external canthus while applying pressure at the internal canthus to prevent drainage of the study drug. Phacoemulsification and limbal paracentesis were performed by an experienced surgeon. Approximately $50 \mu \mathrm{l}$ of $\mathrm{AH}$ was drawn from the anterior chamber with a 30 -gauge needle syringe. After paracentesis, a solution of tropicamide + phenylephrine $\mathrm{HCl}+$ lidocaine $\mathrm{HCl}\left(\right.$ Mydrane $\left.{ }^{\circledR}\right)$ was intracamerally injected to obtain mydriasis and intraocular anaesthesia. Following surgery, patients were treated with antibiotics and/or steroids according to local clinical practice.

The $\mathrm{AH}$ was stored immediately in vials at $-80^{\circ} \mathrm{C}$ and subsequently analysed in a central laboratory. Vial labels contained the study kit number but no information regarding the drug received. The laboratory analyst was blinded to the drug administered, and all samples were analysed for the concentration of three molecules (levofloxacin, dexamethasone sodium phosphate and dexamethasone) by means of a liquid chromatography tandem mass spectrometry method (LC-MS-
MS) developed and validated by the Central Laboratory Ticinumlab (Novara, Italy). Details of the procedure are available in Online Resource 1.

\section{Analytical method}

The analytes were $\mathrm{AH}$ concentrations of levofloxacin, dexamethasone sodium phosphate (DSP) and dexamethasone. A full validation of a specific liquid chromatography tandem mass spectrometry (LC-MS-MS) method quantifying levofloxacin, DSP and its metabolite dexamethasone in human aqueous humour was performed according to a predefined study protocol. Description of the method is available as Online Resource 1.

The analytical procedure was blinded in full GCLP conditions.

\section{Statistical analysis}

Continuous data were summarised with standard descriptive statistics. Categorical data were summarised by frequencies and percentages.

Aqueous humour concentrations of levofloxacin and dexamethasone, measured by LC-MS-MS, were summarised by treatment group, and $95 \%$ confidence limits were provided.

By considering the average volume of the anterior chamber as $161.2 \mu \mathrm{l}$ in $>50$-year-old Caucasian patients as reported by Fan et al. 2019 [10], both the quantity of the analytes present in the anterior chamber at the time of sampling and the fraction of absorbed dose $F$ were calculated. Those data were also summarised by treatment group, and $95 \%$ confidence limits were provided.

The analysis was carried out on the following populations: 
- Per Protocol (PP): all patients who completed the study without any major protocol deviations. Subjects were analysed according to the study treatment they actually received.

- Full Analysis Set (FAS): all randomised patients. According to Intention to Treat (ITT) principles, all patients were analysed considering the randomised treatment assignment.

- Safety (SAF): all patients who received at least one dose of study treatment. Subjects were analysed according to the study treatment they actually received (data not shown).

No formal statistical hypothesis was formulated; all analyses were descriptive in nature, and no inferential statistical tests were planned.

All outputs were produced using SAS® release 9.4 (SAS Institute, Inc., Cary, NC, USA).

\section{Sample size}

The study was to enrol 120 patients, 40 in each treatment group. The sample size was estimated based on the expected precision (width of the $95 \%$ confidence interval) of the estimates of the study drug concentrations:

Dexamethasone When the sample size is 40, a two-sided $95 \%$ confidence interval for a single mean extends $4.648 \mathrm{ng} / \mathrm{ml}$ from the observed mean assuming a standard deviation of $15 \mathrm{ng} / \mathrm{ml}$ and a confidence interval based on large sample $z$ statistic.

Levofloxacin When the sample size is 40 , a two-sided $95 \%$ confidence interval for a single mean extends $61.676 \mathrm{ng} / \mathrm{ml}$ from the observed mean assuming a standard deviation of $199.022 \mathrm{ng} / \mathrm{ml}$ and a confidence interval based on large sample $z$ statistic.

\section{Results}

\section{Patient disposition}

Overall, 133 patients were screened and entered the study treatment between September and December 2018. One patient was instilled a mydriatic agent by error (non-fulfilment of exclusion criteria) and was rescreened in a different occasion for the other eye, making a total of 134 screened patients/eyes. One hundred thirty-one (131) patients $(97.76 \%)$ passed screening and were enrolled. Three (3) patients $(2.24 \%)$ were not enrolled: two were screening failures and one withdrew consent. Six out of the 131 enrolled patients were not randomised: two withdrew the consent; one had punctate keratitis; one had surgery postponed after study completion because a required ECG had not been performed; one, already reported, was instilled a mydriatic agent by mistake and was then rescreened for the other eye; finally, one was not randomised because a freezer for sample storage was not in working order at the due time.

One hundred twenty-five (125) patients (i.e. $95.42 \%$ of screened patients) were randomised: $42(33.60 \%)$ to the levofloxacin + dexamethasone sodium phosphate arm (LDSP), $42(33.60 \%)$ to the levofloxacin arm (L) and 41 $(32.80 \%)$ to the dexamethasone sodium phosphate arm (DSP). All randomised patients received Mydrane ${ }^{\circledR}$ and thus completed the study. Protocol deviations were reported for 9 patients. Three patients $(2.4 \%)$ had been treated with systemic steroids in the 7 days prior to cataract surgery, and 3 received a different drug than the one assigned by randomisation. Two patients received the treatment out of the required time window, and one patient had a history of corneal disease. Those patients did not enter in the PPS.

\section{Demographics and baseline characteristics}

Demographic characteristics of the patient population are detailed in Table 1. Patients were on average 74 years of age, $55.20 \%$ were female and all but three $(97.60 \%)$ were Caucasian. No clinically relevant differences in demographic and background characteristics were found among groups. As expected for patients of this age, the most common ongoing medical conditions were vascular disorders (mainly hypertension: $57.6 \%$ ) and metabolism and nutritional disorders (diabetes mellitus, $18.4 \%$ ).

With regard to ocular medical conditions and surgical history, $8.80 \%$ of patients had an ongoing ocular condition of the eye to be operated, namely $4.76 \%$ of the L-DSP group, $4.76 \%$ of the levofloxacin group and $17.07 \%$ of the DSP group. The most common conditions were exfoliation syndrome $(N=3$, $2.4 \%$ ) (2 patients in the L-DSP group and 1 in the levofloxacin group) and diabetic retinopathy $(N=2,1.60 \%)$ (both in the DSP group).

All patients underwent pachymetry, and all presented corneal thickness within range. Mean corneal thickness was $531.83 \pm 31.26 \mu \mathrm{m}$ in the L-DPS group, $526.50 \pm 30.84 \mu \mathrm{m}$ in the levofloxacin group and $527.29 \pm 34.55$ in the DPS group, respectively. All eyes scheduled for surgery presented intact corneas at the fluorescein test (Oxford scheme grade 0 ).

\section{Analytical method validation}

The analytical method was fully validated for selectivity, linearity, within and between precision, and accuracy. LLOQ was $0.014 \mathrm{nmol} / \mathrm{ml}$ for levofloxacin, $0.058 \mathrm{nmol} / \mathrm{ml}$ for DSP and $0.013 \mathrm{nmol} / \mathrm{ml}$ for dexamethasone. Matrix effect was excluded in blank matrix spiked with analytes. Matrix effect was 
Table 1 Demographic characteristics of the patient population (FAS)

\begin{tabular}{lllll}
\hline & & $\begin{array}{l}\text { L-DSP } \\
(N=42)\end{array}$ & $\begin{array}{l}\text { L } \\
(N=42)\end{array}$ & $\begin{array}{l}\text { DSP } \\
(N=41)\end{array}$ \\
\hline \multirow{2}{*}{ Age (years) } & Mean & 72.45 & 75.38 & 74.59 \\
& SD & 7.60 & 8.38 & 8.00 \\
Gender $N(\%)$ & Range, min-max & $55-88$ & $48-87$ & $57-88$ \\
& Male & $15(35.7 \%)$ & $21(50 \%)$ & $20(48.8 \%)$ \\
Ethnics & Female & $27(64.3 \%)$ & $21(50 \%)$ & $21(51.2 \%)$ \\
& Caucasian & $42(100 \%)$ & $40(95.2 \%)$ & $40(97.6 \%)$ \\
Pachymetry & Black or African American & 0 & $1(2.4 \%)$ & 0 \\
& Asian & 0 & $1(2.4 \%)$ & $1(2.4 \%)$ \\
& Mean & 531.83 & 526.50 & 527.29 \\
& SD & 31.26 & 30.84 & 34.55 \\
& Range, min-max & $455-595$ & $451-581$ & $460-596$ \\
\hline
\end{tabular}

Treatment groups: $L$-DSP levofloxacin + dexamethasone sodium phosphate, $L$ levofloxacin, $D S P$ dexamethasone sodium phosphate also excluded in presence of oxybuprocaine, iodopovidone and benzalkonium chloride, the drugs co-administered with either test or references.

The validation details are available as Online Resource 2.

\section{AH concentrations}

The results of the aqueous humour analysis in the PPS were as follows (details in Table 2 and Fig. 2):

- Levofloxacin's average concentration was $1.970 \mathrm{nmol} / \mathrm{ml}$ (95\% CI $(1.648 ; 2.292))$ in the L-DSP group (corresponding to $711.899 \mathrm{ng} / \mathrm{ml}(95 \%$ CI $595.538 ; 828.260))$ and $2.151 \mathrm{nmol} / \mathrm{ml}(95 \% \mathrm{CI}(1.708 ; 2.594))$ in the levofloxacin group (corresponding to $777.307 \mathrm{ng} / \mathrm{ml}$ (95\% CI 617.220; 937.394)). Levofloxacin was also detected in two patients assigned to the DSP arm
( $0.014 \mathrm{nmol} / \mathrm{ml}$ and $0.193 \mathrm{nmol} / \mathrm{ml}$ respectively), but this could not be attributed to a specific cause. The samples were retested, and the one previously containing $0.014 \mathrm{nmol} / \mathrm{ml}$ (very near to LLOQ) no longer presented trace of levofloxacin.

- Dexamethasone average concentration was $0.030 \mathrm{nmol} /$ $\mathrm{ml}(95 \% \mathrm{CI}(0.025 ; 0.035))$ in the L-DSP group (corresponding to $11.774 \mathrm{ng} / \mathrm{ml}(95 \% \mathrm{CI} 9.812 ; 13.736))$ and $0.042 \mathrm{nmol} / \mathrm{ml}(95 \% \mathrm{CI}(0.035 ; 0.048))$ in the DSP group (corresponding to $16.483 \mathrm{ng} / \mathrm{ml}$ (95\% CI 13.736; 18.838)). Dexamethasone sodium phosphate was not detected in any AH sample, most likely due to its full hydrolysis to free dexamethasone.

One vial containing aqueous humour was damaged and did not allow the analysis of AH drug concentrations for a patient
Table 2 Aqueous humour concentrations $(\mathrm{nmol} / \mathrm{ml})$ - observed values (PPS)

\begin{tabular}{|c|c|c|c|c|c|c|}
\hline & \multicolumn{3}{|c|}{ Concentration of levofloxacin $(\mathrm{nmol} / \mathrm{ml})$} & \multicolumn{3}{|c|}{ Concentration of dexamethasone $(\mathrm{nmol} / \mathrm{ml}$} \\
\hline & $\begin{array}{l}\text { L-DSP } \\
(N=39)\end{array}$ & $\begin{array}{l}\mathrm{L} \\
(N=40)\end{array}$ & $\begin{array}{l}\text { DSP } \\
(N=37)\end{array}$ & $\begin{array}{l}\text { L-DSP } \\
(N=39)\end{array}$ & $\begin{array}{l}\mathrm{L} \\
(N=40)\end{array}$ & $\begin{array}{l}\mathrm{DSP} \\
(N=37)\end{array}$ \\
\hline Mean & 1.970 & 2.151 & 0.006 & 0.030 & 0.000 & 0.042 \\
\hline SD & 0.99 & 1.39 & 0.03 & 0.02 & 0.00 & 0.02 \\
\hline LL CI 95\% & 1.648 & 1.708 & -0.005 & 0.025 & - & 0.035 \\
\hline UL CI 95\% & 2.292 & 2.594 & 0.016 & 0.035 & - & 0.048 \\
\hline Min & 0.777 & 0.347 & 0.000 & 0.000 & 0.000 & 0.013 \\
\hline Q1 & 1.14 & 1.08 & 0.00 & 0.02 & 0.00 & 0.03 \\
\hline Median & 1.93 & 1.69 & 0.00 & 0.03 & 0.00 & 0.04 \\
\hline Q3 & 2.51 & 3.16 & 0.00 & 0.04 & 0.00 & 0.05 \\
\hline Max & 5.361 & 5.942 & 0.193 & 0.070 & 0.000 & 0.108 \\
\hline
\end{tabular}

Concentrations of dexamethasone sodium phosphate were always under the LLOQ and are not reported Treatment groups: $L-D S P=$ levofloxacin + dexamethasone sodium phosphate, $L$ levofloxacin, $D S P$ dexamethasone sodium phosphate. $L L$ lower limit, $U L$ upper limit, $C I$ confidence interval 
Fig. 2 AH concentrations of levofloxacin (top) and of dexamethasone (bottom) after ocular instillation of levofloxacin + dexamethasone sodium phosphate (LDSP), levofloxacin (L) or dexamethasone sodium phosphate (DSP). See doses in the text. Data are means $\pm \mathrm{SD}$;

Nobs $=$ number of observations

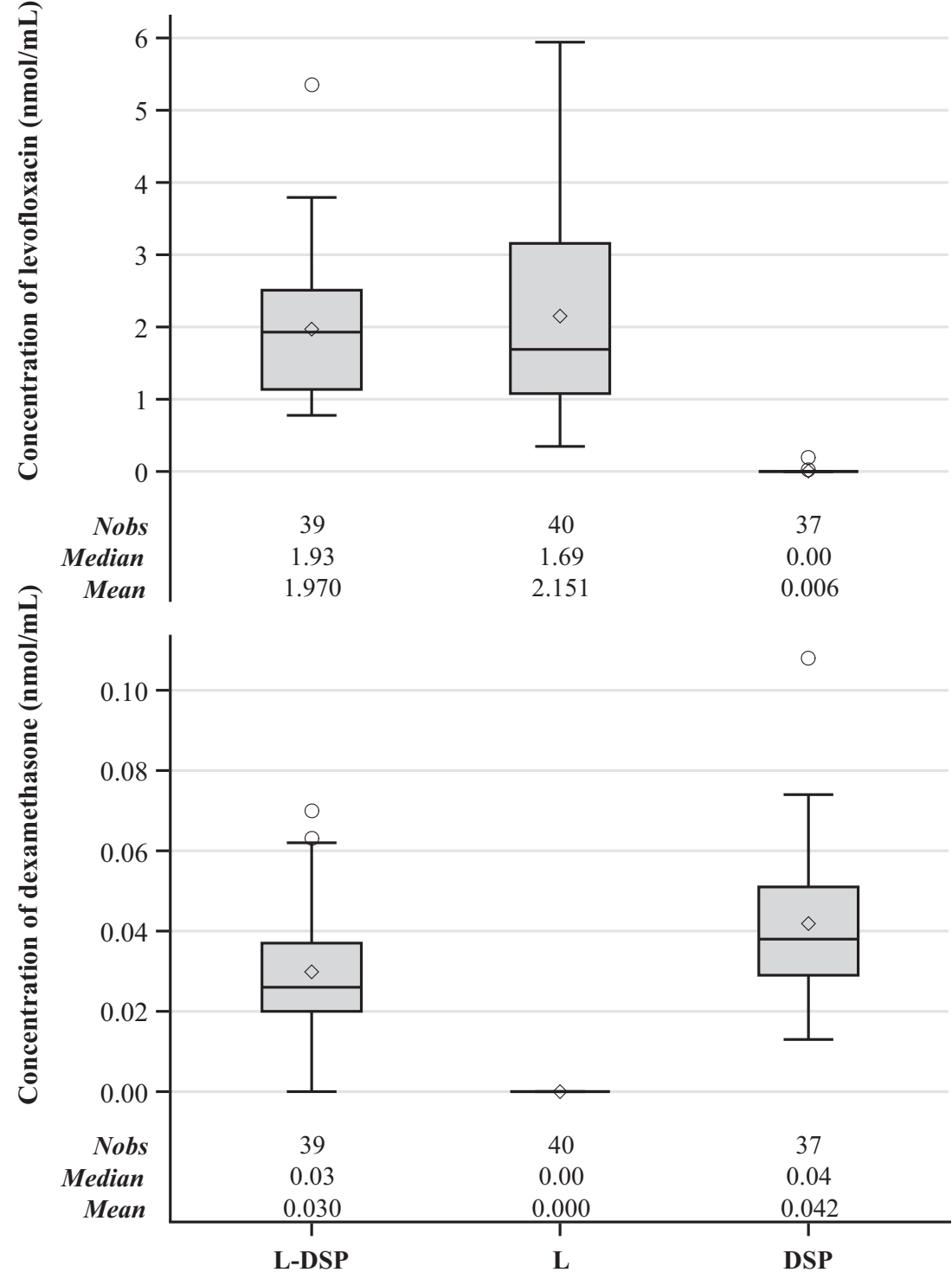

allocated to the L-DSP group. This non-protocol deviation led to the patient's exclusion from the per protocol set but not from the safety set.

\section{QC samples}

The back-calculated concentrations in the in-house control samples were within $\pm 15 \%$ of the nominal value for all samples but one concentration of levofloxacin, which was $+20 \%$ of the nominal value (data not shown).

\section{Fraction $F$ of dose absorbed in the anterior chamber}

The fraction $F$ of dose absorbed in the anterior chamber at the time of AH withdrawal is reported in Table 3. F of levofloxacin was $3.8 \pm 1.9 \cdot 10^{-4}$ after instillation of L-DSP and $4.2 \pm 2.7 \cdot 10^{-4}$ after instillation of $\mathrm{L}$ alone (between groups difference not significant). The $F$ was lower for dexamethasone both after instillation of L-DSP $\left(0.3 \pm 0.2 \cdot 10^{-4}\right)$ and of DSP alone $\left(0.4 \pm 0.2 \cdot 10^{-4}\right)$.

\section{Ratio between MICs and AH levofloxacin concentrations}

The mean concentration of levofloxacin in L-DSP group corresponded to $711.899 \mathrm{ng} / \mathrm{ml}$ (95\% CI 595.538; 828.260). The aqueous humour concentrations were compared with the minimum inhibitory concentrations (MIC) reported by the most recent in vitro study on ocular isolates [11]. The ratios for each strain are reported in Table 4. The actual concentrations of levofloxacin in aqueous humour abundantly exceeded the MICs in the majority of the in vitro-tested strains, with the only exception of MSSA and of some Enterococcus spp. 
Table 3 Fraction $F$ of dose absorbed in the anterior chamber at the time of $\mathrm{AH}$ withdrawal (data $\left.\cdot 10^{-4}\right)$ - observed values (PPS)

\begin{tabular}{|c|c|c|c|c|}
\hline & \multicolumn{2}{|c|}{ Dexamethasone $F$} & \multicolumn{2}{|c|}{ Levofloxacin $F$} \\
\hline & $\begin{array}{l}\text { L-DSP } \\
(N=39)\end{array}$ & $\begin{array}{l}\text { DSP } \\
(N=37)\end{array}$ & $\begin{array}{l}\text { L-DSP } \\
(N=39)\end{array}$ & $\begin{array}{l}\mathrm{L} \\
(N=40)\end{array}$ \\
\hline Mean & 0.3 & 0.4 & 3.8 & 4.2 \\
\hline SD & 0.2 & 0.2 & 1.9 & 2.7 \\
\hline LL CI $95 \%$ & 0.3 & 0.4 & 3.2 & 3.3 \\
\hline UL CI $95 \%$ & 0.4 & 0.5 & 4.5 & 5.0 \\
\hline Min & 0.0 & 0.1 & 1.5 & 0.7 \\
\hline Q1 & 0.2 & 0.3 & 2.2 & 2.1 \\
\hline Median & 0.3 & 0.4 & 3.7 & 3.3 \\
\hline Q3 & 0.4 & 0.5 & 4.9 & 6.1 \\
\hline Max & 0.7 & 1.2 & 10.4 & 11.5 \\
\hline
\end{tabular}

Treatment groups: $L-D S P$ levofloxacin + dexamethasone sodium phosphate, $L$ levofloxacin, $D S P$ dexamethasone sodium phosphate. $L L$ lower limit, $U L$ upper limit, $C I$ confidence interval

\section{Safety}

Only one treatment-emergent adverse event was reported: mild mydriasis in the DSP alone arm.

\section{Discussion}

The combination of dexamethasone sodium phosphate, corresponding to dexamethasone $1 \mathrm{mg} / \mathrm{ml}$, plus levofloxacin $5 \mathrm{mg} / \mathrm{ml}$ (L-DSP) tested in this study is the first combination of a steroid and levofloxacin under development as eye drops. The administration schedule ( -90 and -60 min before paracentesis) was an acceptable compromise between the aim to reduce as least as possible the discomfort to the patient, before the scheduled surgery, and the need to achieve the best approximation to the $\mathrm{C}_{\max }$ of either drug. According to previous data, the peak of levofloxacin concentrations in aqueous humour occurs $2 \mathrm{~h}$ after instillation, with a very small difference between levofloxacin concentrations at 60 and $120 \mathrm{~min}$ [12]. On the other hand, dexamethasone levels in the aqueous humour peak between 90 and 150 min after ocular instillation [4]. Repeated administrations are a common procedure in such studies $[12,13]$, and the choice of the two doses at -90 and $60 \mathrm{~min}$ aimed both to minimise the probability of levels under the LLOQ and to approximate the $t_{\max }$ for both analytes. The late administration of oxybuprocaine and of povidone-iodine (at least 80 and $50 \mathrm{~min}$ after the first and second drop, respectively) would not have affected the absorption of test or
Table 4 Ratio between aqueous humour concentrations in patients after ocular instillation of L-DSP (levofloxacin + dexamethasone sodium phosphate) and in vitro MICs of levofloxacin on ocular isolates (after Yamaguchi et al. $2012^{25}$, modified)

\begin{tabular}{llll}
\hline Bacterial strain & $\begin{array}{l}\text { No. } \\
\text { isolates }\end{array}$ & $\begin{array}{l}\text { MIC90 } \\
(\mu \mathrm{g} / \mathrm{l})\end{array}$ & $\begin{array}{l}\text { AH concentrations } \\
\text { /MIC90 ratio LL-UL } \\
95 \% \text { CI }\end{array}$ \\
& & & $297-414$ \\
\hline Streptococcus pyogenes & 434 & 2.0 & $596-828$ \\
Streptococcus pneumoniae & 661 & 1.0 & $1191-1657$ \\
Methicillin-susceptible Staphylococcus aureus & 745 & 0.5 & $149-207$ \\
MSSA: Methicillin-susceptible coagulase-negative & 557 & 4.0 & \\
$\quad$ Staphylococci & 719 & $>64$ & $<9.3-<12.9$ \\
MRSA: Methicillin-resistant Staphylococcus aureus & 732 & 16.0 & $37.2-51.8$ \\
Methicillin-resistant coagulase-negative Staphylococci & 641 & 32 & $18.6-25.88$ \\
Enterococcus faecalis & 591 & 64 & $9.3-12.9$ \\
Enterococcus faecium & 566 & 0.06 & $9926-13,804$ \\
Moraxella catarrhalis & 80 & 16 & $37.2-51.8$ \\
Neisseria gonorrhoeae & 741 & 16 & $37.2-51.8$ \\
Escherichia coli & 678 & 0.5 & $1191-1657$ \\
Klebsiella pneumoniae & 603 & 1.0 & $596-828$ \\
Citrobacter spp. & 657 & 0.5 & $1191-1657$ \\
Enterobacter spp. & 590 & 8.0 & $74.44-104$ \\
Proteus mirabilis & 521 & 0.5 & $1191-1657$ \\
Indole-positive Proteus spp. & 650 & 2.0 & $297-414$ \\
Serratia marcescens & 194 & 0.125 & $4764-6626$ \\
Salmonella spp. & 660 & 0.03 & $19,851-27,609$ \\
Haemophilus influenzae & 577 & 4.0 & $149-207$ \\
Acinetobacter spp. & 609 & 64 & $9.3-12.9$ \\
Pseudomonas aeruginosa UTI & 860 & 8.0 & $74.44-104$ \\
Pseudomonas aeruginosa RTI & & & \\
\hline & & & \\
\hline
\end{tabular}

$L L$ lower limit, $U L$ upper limit, $C I$ confidence interval 
reference drugs, and the matrix effect was excluded during the validation study, as detailed in Online Resource 2.

The mean concentration of levofloxacin in L-DSP group corresponded to $711.899 \mathrm{ng} / \mathrm{ml}(95 \%$ CI 595.538; 828.260), and the fraction $F$ of levofloxacin dose absorbed in the anterior chamber at the time of $\mathrm{AH}$ withdrawal was $3.8 \cdot 10^{-4}$. There was a minimum difference ( $8 \%$ lower, not relevant) in the aqueous humour concentration of levofloxacin in the LDSP arm compared with levofloxacin alone arm. In order to understand the clinical relevance of that result, the aqueous humour concentrations at the investigated time (supposed to correspond to the $\mathrm{AH}_{\mathrm{max}}$ ) have been compared with the minimum inhibitory concentrations (MIC) reported by the most recent in vitro study on ocular isolates [11]. It is evident that the actual concentrations of levofloxacin in aqueous humour abundantly exceeded the MICs in the majority of the in vitro-tested strains, with the only exception of MSSA and of some Enterococcus spp. The ESCRS Guidelines for Prevention and Treatment of Endophthalmitis Following Cataract Surgery [1] underline a number of studies confirming that a concentration/MIC ratio above approximately 30 (for many gram-positive strains), and above 100 (for gramnegative) was needed to eradicate bacteria. Our study confirmed that levofloxacin concentrations well above the MICs are found after ocular instillation of the test product, with $\mathrm{AH}$ concentration/MIC ratio exceeding the threshold of 30 in gram-positive and of 100 in gram-negative bacteria in the majority of the isolated strains.

Dexamethasone sodium phosphate concentrations in aqueous humour resulted to be under the LLOQ for all specimens. Free dexamethasone levels were measurable in almost all $\mathrm{AH}$ specimens, showing that the DSP was hydrolysed to the active metabolite in all samples. The aqueous humour concentrations of dexamethasone were equal to $0.030 \mathrm{nmol} / \mathrm{ml}(95 \% \mathrm{CI}$ $(0.025 ; 0.035))$ in the L-DSP arm (corresponding to $11.774 \mathrm{ng} / \mathrm{ml}(95 \%$ CI 9.812; 13.736)) and slightly larger in the single-agent arm. The fraction $F$ of dexamethasone dose absorbed was about $0.3 \cdot 10^{-4}$, less than one tenth of that of levofloxacin. Nevertheless, the concentrations are great enough to carry out their expected pharmacological activity. If we consider that oral dexamethasone as an antiinflammatory agent in ocular conditions is approved for a 2mg daily dose [14], and that the volume of distribution at the steady state $\mathrm{V}_{\mathrm{ss}}$ is about $1 \mathrm{l} / \mathrm{kg}$ in women, one half in men [15], assuming an $81 \%$ bioavailability [16] and a homogeneous distribution, then the quantity of dexamethasone reaching the anterior chamber $(161.2 \mu \mathrm{l})$ after systemic administration would theoretically be about $0.011 \mathrm{nMoles}$, i.e. the same order of magnitude as the dexamethasone amount measured in the anterior chamber in our study. The ocular instillation of DSP is very efficient in this respect, and this was confirmed by the pivotal investigation of L-DSP in a controlled study [17] versus tobramycin and dexamethasone eye drop suspension, which was considered up to now the gold standard for topical treatment in the intended indication.

Notably, aqueous humour concentrations of dexamethasone were slightly lower in the L-DSP combination arm than in the single-agent DSP arm. Rather than the effect of a pharmacokinetic interaction between the two active ingredients of the combination product, the slightly larger penetration of dexamethasone in the $\mathrm{AH}$ of patients in the reference group may have been due to the higher concentration of the active ingredient in the commercial preparation. In fact, it is wellknown that the most important mechanism of elimination of active ingredients from the cul-de-sac is drainage through tear fluid, which may have been more efficient in the presence of a larger volume of eye drops instilled for the test product compared with the reference drug. DSP was not detected in the samples of any of the three treatment groups, even when DSP was part of the instilled dose, most likely due to its full hydrolysis to free dexamethasone.

Since the study purpose was to evaluate the penetration of levofloxacin and dexamethasone sodium phosphate into the $\mathrm{AH}$ rather than to compare treatments groups, the sample size was based on the precision of the estimate, and no formal tests were performed to assess whether any differences in arms were statistically significant.

Postoperative inflammation usually is self-limiting, but steroidal drugs are often prescribed to prevent and treat postoperative inflammation, shortening recovery time and improving ocular comfort. The combined administration of a corticosteroid and an antibiotic provides a powerful anti-inflammatory effect, and at the same time a preventive and therapeutic action against infections.

Combined eye drops are comfortable for patients and improve medication compliance. This is of great importance in elderly persons, who represent at least $80 \%$ of the target population of L-DSP. Moreover, FDCs are superior to extemporaneous combinations of different products, as the FDCs are guaranteed by chemical-pharmaceutical investigation as well as by preclinical and clinical studies. Not only the efficacy and safety in humans but also the non-clinical toxicity profile and safety margin for human use are carefully investigated for FDCs. On the contrary, extemporaneous combinations are mostly based on empirical use, and their risk for patients may be underestimated. FDCs also favour the proper administration of the two agents, reduce the possibility of inaccurate dosage and improve patient compliance with medication. Finally, the use of a FDC investigated in a well-designed absorption study would avoid the reciprocate dilution in the cul-desac of empirically combined active ingredients and possible consequent decrease of efficacy of either product.

In conclusion, our results confirm that no interaction is evident on the corneal penetration of levofloxacin and dexamethasone, which reach pharmacologically active 
concentrations when instilled as fixed combination eye drops to patients undergoing cataract surgery.

Acknowledgements Analytics of the active ingredients and metabolites in the aqueous humour were performed in Ticinumlab, Novara, Italy. The contribution of Luisa Zangirolami, Giulia Martinetto and Matteo Avonda from Ticinumlab is acknowledged. Randomisation list, monitoring of clinical centres, project management and regulatory activities, data management and statistics were provided by OPIS, Desio, Italy. The contribution of Valentina Belloni as Trial Leader and of Giulia Litta Modignani as Project Manager, and of Chiara Costantini and Veronica Pagano as statisticians from OPIS is acknowledged.

Contributions of authors statement Michele Figus designed the study and analysed the results. Marco Nardi and Luca Rossetti performed the research as principal investigators of the respective centres. Michele Figus, Chiara Posarelli and Dario Romano operatively contributed to the research performance. The first draft of the manuscript was written by Chiara Posarelli, and all authors commented on previous versions of the manuscript. All authors read and approved the final manuscript.

Data availability statement All data generated or analysed during this study are included in this published article.

Funding information The research was funded by NTC srl, Milan - Italy, owner of the developmental levofloxacin and dexamethasone eye drops solution.

\section{Compliance with ethical standards}

Conflict of interest The authors declare that they have no conflict of interest.

Open Access This article is licensed under a Creative Commons Attribution 4.0 International License, which permits use, sharing, adaptation, distribution and reproduction in any medium or format, as long as you give appropriate credit to the original author(s) and the source, provide a link to the Creative Commons licence, and indicate if changes were made. The images or other third party material in this article are included in the article's Creative Commons licence, unless indicated otherwise in a credit line to the material. If material is not included in the article's Creative Commons licence and your intended use is not permitted by statutory regulation or exceeds the permitted use, you will need to obtain permission directly from the copyright holder. To view a copy of this licence, visit http://creativecommons.org/licenses/by/4.0/.

\section{References}

1. Barry P, Cordovés L, Gardner S ESCRS Guidelines for prevention and treatment of endophthalmitis following cataract surgery: data, dilemmas and conclusions 2013. https://www.escrs.org/downloads/ Endophthalmitis-Guidelines.pdf. last accessed 7 Sept 2019

2. Behndig A, Cochener B, Guell JL et al (2013) Endophthalmitis prophylaxis in cataract surgery: overview of current practice patterns in 9 European countries. J Cataract Refract Surg 39:14211431
3. Chang DF, Braga-Mele R, Handerson BA, Mamalis N, Vasavada A, ASCRS Cataract Clinical Commitee (2015) Antibiotic prophylaxis of postoperative endophthalmitis after cataract surgery: results of the 2014 ASCRS member survey. J Cataract Refract Surg 41(6): $1300-1305$

4. Cagini C, Cometa F, Torroni G, Pellegrino A, Pellegrino R, Cavallini GM (2016) Dexamethasone disodium phosphate penetration in to the human aqueous humour after topical application. Curr Eye Res 41(7):897-899

5. Bucci FA (2004) An in vivo study comparing the ocular absorption of levofloxacin and ciprofloxacin prior to phacoemulsification. Am J Ophthalmol 137:308-312

6. Healy DP, Holland EJ, Nordlund ML, Dunn S, Chow C, Lindstrom RL, Hardten D, Davis E (2004) Concentrations of levofloxacin, ofloxacin, and ciprofloxacin in human corneal stromal tissue and aqueous humour alter topical administration. Cornea. 23:255-263

7. Puustjarvi T, Terasvirta M, Nurmenniemi $P$ et al (2006) Penetration of topically applied levofloxacin $0.5 \%$ and ofloxacin $0.3 \%$ into the vitreous of the non-inflamed human eye. Graefes Arch Clin Exp Ophthalmol 244:1633-1637

8. Yamada M, Mochizuki H, Yamada K et al (2002) Aqueous humour levels of topically applied levofloxacin in human eyes. Curr Eye Res 24:403-406

9. Rosenblum C, Dengler RE Jr, Geoffroy RF (1967) Ocular absorption of dexamethasone phosphate disodium by the rabbit. Arch Ophthalmol 77(2):234-237

10. Fan S, Guo T, Chen B et al (2019) Differences in ocular biometrics and aqueous humour dynamics between Chinese and Caucasian adults. Br J Ophthalmol 20

11. Yamaguchi K, Ohno A, Ishii Y, Tateda K, Iwata M, LevofloxacinSurveillance Group (2012) In vitro susceptibilities to levofloxacin and various antibacterial agents of 12,866 clinical isolates obtained from 72 centers in 2010. Jpn J Antibiot 65(3):181-206 Translation from Japanese

12. Bucci FA Jr, Nguimfack IT, Fluet AT (2016) Pharmacokinetics and aqueous humor penetration of levofloxacin $1.5 \%$ and moxifloxacin $0.5 \%$ in patients undergoing cataract surgery. Clin Ophthalmol 10: 783-789

13. Wejitens O, Schoemaker RC, Romijn FP et al (2002) Intraocular penetration and systemic absorption after topical application of dexamethasone disodium phosphate. Ophthalmology 109(10): 1887-1891

14. Decadron - Riassunto delle caratteristiche del prodotto (SmPC) Italy, from AIFA website https://farmaci.agenziafarmaco.gov.it/ bancadatifarmaci/farmaco?farmaco $=014729$. Accessed 31 Mar 2020

15. Berezhkovskiy LM (2013 Feb) Prediction of drug terminal half-life and terminal volume of distribution after intravenous dosing based on drug clearance, steady-state volume of distribution, and physiological parameters of the body. J Pharm Sci 102(2):761-771

16. Spoorenberg SM, Deneer VH, Grutters JC, Pulles AE, Voorn GP, Rijkers GT, Bos WJ, van de Garde EM (2014 Jul) Pharmacokinetics of oral vs. intravenous dexamethasone in patients hospitalized with community-acquired pneumonia. Br J Clin Pharmacol 78(1):78-83

17. Coassin M, Bandello F Medical treatment after cataract surgery: evidence for a new anti-inflammatory strategy. Proc. 37th Congress of the ESCRS, Paris, 2019, FP-297744

Publisher's note Springer Nature remains neutral with regard to jurisdictional claims in published maps and institutional affiliations. 